\title{
Didáctica para la formación de investigadores desde la investigación vivencialista - intuitiva
}

\author{
Didactics for the training of researchers from the \\ experientialist - intuitive research
}

\section{Carretero Valdez, Sandra Verónica}

Dandra Verónica Carretero Valdez sandracarretero@hotmail.es

Universidad Autónoma Tomás Frías, Bolivia

\section{Delectus}

Instituto Nacional de Investigación y Capacitación Continua, Perú ISSN-e: 2663-1148

Periodicidad: Semestral

vol. 2, núm. 2, 2019

publicaciones.iniccperu@gmail.com

Recepción: 17 Julio 2019

Aprobación: 13 Diciembre 2019

Publicación: 28 Diciembre 2019

URL: http://portal.amelica.org/ameli/journal/390/3902683001/

Esta licencia permite a otros entremezclar, ajustar y construir a partir de su obra con fines no comerciales, y aunque en sus nuevas creaciones deban reconocerle su autoría y no puedan ser utilizadas de manera comercial, no tienen que estar bajo una licencia con los mismos términos.

\section{c) (7) (8)}

Esta obra está bajo una Licencia Creative Commons AtribuciónNoComercial 4.0 Internacional.
Resumen: El artículo describe el proceso investigativo que se desarrolló con un grupo de investigado- res nóveles en la Facultad de Ciencias Sociales y Humanísticas-Trabajo Social-Sede Uncía de la Universidad Autónoma Tomás Frías durante la gestión 2018. Su propósito fue impul- sar un proceso alternativo en la formación de trabajadores sociales para investigar, com- prender y transformar su objeto de estudio e intervención: los problemas sociales al interior de las familias de la comunidad. Corresponde a una investigación cualitativa desde la teoría vivencialistaintuitiva; al brindar al estudiante la posibilidad de intervenir sobre problemas concretos, aplicando técnicas de investigación científica, la característica fundamental fue partir de la cercana vivencia de algunos hechos abstraídos de la realidad de cada familia para luego ser proyectados en niveles de percepción cognitiva superiores. Como resulta- do, los estudiantes lograron acercarse a la realidad concreta, conocer los problemas que aquejan a las familias, comprender su realidad y con ello encarar un proceso investigativo involucrando a las personas como actores sociales capaces de asumir un rol protagónico en la transformación de su realidad.

Palabras clave: Didáctica, formación de investigadores, investigación vivencialista-intuitiva.

Abstract: The article describes the investigative process that
was developed with a group of novice researchers in the
Faculty of Social and Humanistic Sciences-Social Work- Uncia
Head- quarters of the Tomas Frias Autonomous University
during the 2018 management. Its pur- pose was to promote
an alternative process in the training of social workers, in
order to investigate, understand and transform their object
of study and intervention: social pro- blems inside families'
community. It corresponds to a qualitative investigation from
the experientialist-intuitive theory; by giving the student the
possibility to interfere on specific problems, applying scientific
research techniques, the fundamental characteristic was to start
from the close experience of some facts abstracted from the
reality of each family and then be projected at higher levels
of cognitive perception. As a result, the students managed to
get closer to the concrete reality, know the problems that
afflict the families, understand their reality and with it to
face an investigative process involving people as social actors
capable of assuming a leading role in the transformation of


their reality. 1. UNIVERSIDAD AUTÓNOMA TOMÁS FRÍAS, Bolivia | sandracarretero@hotmail.es | https:// orcid.org/0000-0001-5448-0554

Keywords: Didactic, training of researchers, experientialistintuitive research.

\section{INTRODUCCIÓN}

A nivel internacional los procesos de formación de investigadores van cobrando notoria importancia. La necesidad de contar con docentes investigadores en las universidades implica la apropiación del método científico que permita la generación de conocimiento mediante los procesos investigativos.

Ante esta realidad, dos cuestiones salen a relucir, la primera referida a la necesidad de comprender lo que es investigación; premisa planteada por Padrón (2018) donde plantea: "si no se comprende con precisión lo que es investigar, ni la estructura ni funcionalidad de los procesos de investigación científica, entonces nunca se podrá investigar y mucho menos formar investigadores". Esta última afirmación, permite considerar el otro asunto, la formación la enseñanza de la investigación.

Ello da pie al análisis de lo que de ordinario desarrollamos en las aulas universitarias en el área de la investigación, como uno de las funciones fundamentales de la universidad y educación superior en general. Función asumida por diferentes niveles de formación, pero poco aplicada -y al parecer muy poco comprendida- en su verdadera dimensión.

En el mundo siempre va existir hechos, procesos observables que necesitan ser explicados para generar conocimiento; la investigación científica posibilita estas explicaciones y respuestas a problemas surgidos en la realidad.

En los espacios académicos se utiliza la metodología de la investigación como punto de partida para desarrollar ciertos procesos investigativos, entendida como:

Un conjunto de procedimientos recetas predefinidas que deben ser seguidas al pie de la letra, carecen de razonamiento de teorización, sobre la base que dicen los autores, sin embargo, se convirtió en uno de los obstáculos para el aprendizaje de la investigación, por ello no es una referencia para realizar investigaciones; nadie puede enseñar a investigar si no lo ha hecho... (Padrón, 2018).

Idea reafirmada en diversos espacios de discusión académica por Fernández (2018)uno no puede exigir lo que no puede dar...se debe enseñar a investigar investigando.

Quienes enseñan investigación reducen sus acciones didácticas a centrar la atención en manuales o escritos sobre la metodología de investigación, tal como si ello garantizaría la generación de producción investigativa. Poco preocupa la particularidad que reviste la enseñanza de la investigación.

Entonces ¿`cómo enseñar a investigarr? En la perspectiva de Sánchez (2014), lograr una didáctica distinta de la investigación científica, implica:

Enseñar a investigar teniendo como referente el proceso mismo de la generación efectiva de conocimiento científico...Si se quiere enseñar a investigar prácticamente, es decisivo cambiar el énfasis de la didáctica de la investigación, pasando de una enseñanza teórica, abstracta y general a una didáctica práctica, basada en la capacitación y el entrenamiento en las operaciones que ocurren real y efectivamente durante el proceso de la producción de conocimiento científico (p. 20).

Un formador de investigadores debe comprender -y no solo conocer- la generación de conocimiento científico, asumir determinada posición investigativa en cuanto al estilo de pensamiento o el enfoque epistemológico que reafirme el paradigma investigativo. Complementario a ello, discernir la tecnología didáctica más apropiada al estudiante en su realidad y contexto socio-cultural, apropiarse de estas ideas es el desafío continuo para la enseñanza de la investigación y el aprendizaje consecuente del estudiante. 
De ahí que la posibilidad de encarar un proceso investigativo a partir del referente teórico de la teoría de investigación vivencialista, se constituye en una experiencia diferente para la realidad en el que se desenvuelven los estudiantes que más allá de un aprendizaje teórico permite una experiencia real, pues posibilita un elemento vital para el estudiante de trabajo social, la convivencia y comprensión de realidades sociales concretas. La teoría vivencialista como tecnología de investigación en sus fundamentos más profundos plantea un proceso de vivencia en el contexto donde se suceden los hechos en lo exterior, lo que se observa; pasa a un plano de discernimiento de reflexión interior y luego a un nivel superior que en este caso es la comprensión de la realidad que debe ser transformada (Padrón, 2014).

Son estos referentes teóricos los que posibilitaron la experiencia de desarrollar procesos investigativos en un contexto familiar cuyas realidades sociales se prestan al estudio de problemas sociales concretos diversos.

De estas consideraciones la pregunta que sirvió de base para la investigación estuvo centrada en ¿Cómo generar la formación de investigadores en el campo del trabajo social, desde la investigación vivencialistaintuitiva? En este propósito el objetivo de la investigación, Formar investigadores en el campo del trabajo social, desarrollando un proceso alternativo para la enseñanza y el aprendizaje de la investigación desde la teoría vivencialista-intuitiva que permita comprender y transformar la realidad.

El argumento fundamental de este estudio es, la inmersión en los hechos, a la realidad concreta de las familias, permite la abstracción de conocimiento y de ahí a transformar situaciones concretas. Esto conduce a generar ciencia mediante la producción de investigación.

Toda investigación está conformada por dos componentes: hechos concretos observables y las explicaciones, respuestas a estos problemas. De ahí, que toda teoría de la investigación conlleva un componente fáctico y uno abstracto, formulación mental que explica los hechos.Resulta entonces, importante identificar esos hechos y saber agruparlos coherentemente para estudiarlos; los criterios para ello, como patrones, estilos o formas de dar explicaciones conllevan diferentes connotaciones, como la determinación de la teoría de investigación que permite la claridad del estilo de pensamiento o enfoque epistemológico asumido por el investigador. Se parte por asumir la teoría especial de investigación vivencialista, que considera la importancia de la inmersión en los hechos, vivir, experimentar, intuir será lo que conduzca a generar ciencia y teoría de la investigación. El paradigma socio-crítico es entonces la concepción global que abriga la investigación, bajo los siguientes referentes. 
TABLA 1.

Aspectos metodológicos de la investigación desde el paradigma sociocrítico.

\begin{tabular}{|c|c|c|}
\hline CARACTERÍSTICAS & $\begin{array}{l}\text { PARADIGMA } \\
\text { SOCIO CRÍTICO }\end{array}$ & $\begin{array}{l}\text { APLICACIÓN EN LA } \\
\text { INVESTIGACIÓN }\end{array}$ \\
\hline Interés & $\begin{array}{l}\text { Emancipar, } \\
\text { criticar e identificar } \\
\text { el potencial para el } \\
\text { cambio. }\end{array}$ & $\begin{array}{l}\text { Desarrollar un proceso } \\
\text { alternativo para la enseñanza } \\
\text { y el aprendizaje de la } \\
\text { investigación, desde la teoria } \\
\text { vivencialista-intuitiva, que } \\
\text { permita comprender y } \\
\text { transformar la realidad. }\end{array}$ \\
\hline $\begin{array}{l}\text { Relación } \\
\text { investigador - } \\
\text { investigado }\end{array}$ & $\begin{array}{l}\text { Sujeto a sujeto } \\
\text { (Relación } \\
\text { democrática, } \\
\text { comunicativa y } \\
\text { propositiva) }\end{array}$ & $\begin{array}{l}\text { Relacionamiento entre: } \\
\text { - Investigadores (18 } \\
\text { estudiantes) } \quad \text { Familias } \\
\text { Municipio de Uncia (18) } \\
\text { - Docente investigador }\end{array}$ \\
\hline Enfoque & Cuali - cuantitativo & Cualitativo \\
\hline Método & $\begin{array}{l}\text { Su tratamiento } \\
\text { de los datos es } \\
\text { cuali-cuantitativo } \\
\text { (dialéctica) }\end{array}$ & $\begin{array}{ll}\text { - } & \text { Fenomenológico } \\
\text { - } & \text { Historias de Vida } \\
\text { - } & \text { Estudio de casos } \\
\text { profundidad } & \text { Observación } \\
\end{array}$ \\
\hline Contexto & $\begin{array}{l}\text { Ambiente natural } \\
\text { espontáneo }\end{array}$ & $\begin{array}{l}\text { Residencia de las familias del } \\
\text { Municipio de Uncia - Potosí - } \\
\text { Bolivia. }\end{array}$ \\
\hline Propósitos & $\begin{array}{l}\text { Revelar los } \\
\text { significados de las } \\
\text { formas } \\
\text { particulares de la } \\
\text { vida social y } \\
\text { emancipación. }\end{array}$ & $\begin{array}{l}\text { Comprender la realidad y los } \\
\text { problemas emergentes en la } \\
\text { cotidianidad de las familias } \\
\text { del Municipio de Uncia. }\end{array}$ \\
\hline Lógica & $\begin{array}{l}\text { Dialéctica entre lo } \\
\text { deductivo e } \\
\text { inductivo. }\end{array}$ & $\begin{array}{l}\text { Partir de la intuición y la } \\
\text { aproximación a realidades } \\
\text { concretas (inducción) y } \\
\text { arribar a generalizaciones } \\
\text { (deducción). }\end{array}$ \\
\hline
\end{tabular}

Adaptado en base a Koetting, 1984 (citado por Arteaga, 2011).

El procedimiento metodológico para la investigación, estuvo centrado en cuatro momentos:

1. Primer momento: Orientaciones didácticas para la formación de investigadores. Aspectos fundamentales

- Contextualización de la investigación, a partir de un taller en aula.

- Aclaración de conceptos básicos.

- Motivación continua a los estudiantes.

- Considerar elementos como: qué realidades se investigarán, porqué y cuál el propósito de investigar esas realidades.

2. Segundo momento: Vivencia, experiencia, relacionamiento, intuición.

- Inmersión en la realidad: identificación y selección de familias que presenten problemas sociales concretos

- Establecer relaciones de cercanía, involucrándose en la realidad de cada familia.

- Generar habilidades para el relacionamiento. Generar habilidades científicas.

- Generar habilidades éticas.

- Acciones que duraron un mes, concluyendo con un diagnóstico preliminar 
3. Tercer momento: Aspectos teórico - metodológicos

- Aclaración y determinación de conceptos básicos a partir de un taller.

- Potenciar el conocimiento adquirido a partir de la vivencia obtenida en el relacionamiento con las familias.

- Identificación, análisis y priorización de los problemas identificados.

- Determinar título, problema, objetivo, métodos, contextualización teórica.

- Categorización del problema.

- Desarrollar los antecedentes de la investigación, establecer argumentaciones, justificación, impacto social, científic de la investigación.

- Revisión bibliográfica que argumente las categorías identificadas, construyendo de esta forma un marco teórico.

- La estrategia para este acápite, la elaboración de esquemas y mapas conceptuales que permitan identificar las principales ideas acerca el tema.

- Los aspectos metodológicos implicaron asumir estrategias como: la selección de técnicas apropiadas con las que se visualicen trabajar los estudiantes; su procesamiento y análisis de datos a partir de la valoración de las habilidades para la investigación.

- De la relación emprendida con las familias, fue fundamental la apertura y confianza para la aplicación de los métodos empíricos como la entrevista a profundidad y la observación que fueron aplicados en varias ocasiones en función a los temas a investigar.

4. Cuarto momento: Orientaciones didácticas para la elaboración del informe de inves- tigación y la socialización.

- Capacitación para el procesamiento y organización de la información.

- Normativas institucionales - académicas a seguir.

- Elaboración del documento final.

- Devolución y socialización de resultados a la población participante, se procedió a la etapa conclusiva y cierre del proceso con cada familia.

- Socialización de resultados como exigencia académica.

La investigación Vivencialista-Intuitiva

Desde la teoría de la investigación resulta importante reconocer los estilos de pensamiento del investigador o enfoques epistemológicos lo que trasunta en los procesos investigativos concretos, claramente diferenciables uno del otro.

La intuición (vivencia, introspección, comprensión), la demostración (razonamiento, argu- mentación, explicación) y la sensación (captación sensorial, observación, instrumentación) constituyen para Locke tres formas elementales de conocimiento correlacionadas con la na- turaleza del objeto conocido, formas que coexisten en el ser humano (equivale a otra tesis según la cual los distintos Estilos de Pensamiento coexisten en una misma personal y varían según la situación de individuo y la naturaleza del problema a resolver) (Padrón, 2014, p. 1).

En esta perspectiva tres son los estilos de pensamiento, según cada uno de ellos la estructura de investigación varía sustancialmente:

a) Empirista - inductivista (sentidos) tipo de investigación medicional de grandes investigadores del siglo XIX; asume como objeto de investigación los hechos ob- servables de la experiencia los datos a través de los sentidos, para que por medio de mecanismo inductivo llegue a la formulación de leyes.

b) Racionalista - deductivista (razón) sustenta que la ciencia y la investigación se consigue a partir de la razón el pensamiento, aunque existiera algo no observable, en tanto pueda ser pensado será susceptible de ser investigado. 
c) Vivencialista - intuitiva (vivencias) reconoce la importancia de la inmersión en los hechos, vivir, experimentar será lo que conduzca a generar ciencia y teoría de la investigación.

El vivencialismo según Hernández (2016)

Son expresiones de la experiencia vital consciente humana. El vocablo vivencialismo provie- ne de la palabra vivencia que es el estado de vivir; así tenemos que vive un perro, vive una planta, pero la única entidad viviente que entiende que vive es el humano, transformándose la vivencia en vivencialista, experiencia que determina la posibilidad de crear los vivencialismos (p.8).

Esta teoría parte por la concepción de una teoría general de la investigación, que implica concebir tendencias de los estilos de pensamiento, dentro los procesos de investigación. La teoría especial vivencialistaexperiencialista se constituye en parte de esta teoría general que enfatiza la vivencia, la experiencia y la intuición como fundamento para los procesos investigativos.

Se inicia con la observación de casos concretos de la realidad, sin embargo, no solo obser- varlos, percibirlos, sino de vivirlos; no se trata de conocer aquello que se encuentre lejos de nosotros, sino de aquellos hechos que puedan ser vividos: se tiene el hecho se abstrae, se observa y se viven esos hechos, lo fundamental es tener la vivencia concreta para producir conocimiento.

La empatía aparece como uno de los conceptos fundamentales, ponerse en el lugar de quie- nes están viviendo determinadas situaciones. La convivencia de igual forma es elemental en esta tecnología investigativa, cuando uno se convierte en parte de aquello que se quiere comprender, cuando las cosas exteriores se asumen, se viven como parte de uno como in-vestigador. La intuición es otro proceso particular, como aquellas cosas que se observa lo que esta externamente, no se trata de inducir o inferir, sino intuir como proceso cognitivo de percibir, descubrir y tender o asumir cierta información su significado como conocimiento científico. Lo importante no es el dato sino la vivencia de ciertos datos.

Postulados de la teoría de investigación Vivencialista-Intuitiva

A diferencia de la teoría empirista inductivista, lo que busca esta teoría es la comprensión antes que la explicación. De las cosas que aparentan ser algo, es necesario ver lo que hay detrás en esencia, su naturaleza, decodificar y llegar a la interpretación más profunda de los hechos y así comprender los hechos de investigación. Entonces la apariencia y la realidad connotan profundas diferencias, para ello es necesario llegar a la esencia lo que obliga a vivir los hechos.

La comprensión, por tanto, es uno de los postulados más importantes de una tecnología vivencialistaintuitiva, comprender a partir del conocimiento de lo que se investiga y se tenga la capacidad de interpretarlo. De igual forma hay diferencia entre objeto y sujeto, la importancia de la subjetividad recobra gran valor. Sin embargo, en tanto estos niveles de subjetividad no llegan a los demás no son capaces de socializarse entonces carecen de niveles de veracidad.

Como tecnología de investigación vivencialista-intuitiva parte de hechos de estudio que deberán ser orientados a los procesos humanos; es decir que sea vivible experienciable. Se debe definir un escenario, ámbito de ocurrencia típica, un espacio donde existen hechos que se desea vivenciar, se deciden categorías de análisis hechos que permitan las vivencias.

Esta tecnología de investigación permite una construcción teórica de lo que se estudia, de ahí se encuentran los procesos hermenéuticos, fenomenológicos, etnográficos, todos los que permiten aportar al conocimiento; los significados más profundos de los hechos serán obtenidos por intuición comola capacidad del investigador de perspicacia, percepción. Las emociones, los valores, el lenguaje, todos los hechos cualitativos son susceptibles de ser investigados mediante el esquema operativo de la tecnología de investigación vivencialistaintuitiva.

Finalmente, la tecnología de investigación vivencialista-intuitiva implica fundamentalmen- te experimentar la vivencia de los fenómenos de estudio, no solo basta observarlos sino deben ser vividos para contar con la capacidad de no solo explicarlo sino y sobre todo comprenderlo, de esta forma se podrá 
interpretar el hecho, pero sobre todo poder socializar el hecho y de esta forma generar conocimiento científico. Esta tecnología, por tanto, no será adecuado para todos los hechos o fenómenos observables tangibles de la realidad del mundo exterior, sino para aquellos referidos o vinculados con los procesos humanos que se pueda vivir, ser parte de esa situación o fenómeno observable y vivible.

Enseñanza y aprendizaje de la investigación

Enseñar a investigar no es tarea fácil, es más bien un proceso complejo y una actividad diversificada. Sánchez (2014) refiere que es complejo:

Pues en la enseñanza de la investigación concurren numerosas operaciones, no menos densas, relativas a 1) lo que se enseña al enseñar a investigar, y 2) cómo se enseña a investigar. Estos dos tipos de operaciones constituyen dos prácticas distintas, la de producir conocimiento nuevo y la de enseñar a producirlo. La primera conforma el oficio de investigador; la segunda, el oficio de pedagogo... (2014, pp. 12-13).

No tener claridad de estas dos puntualizaciones es un error recurrente en diversas realidades en el ámbito universitario.

Padrón (2004) en una especie de metáfora frente a la realidad de la enseñanza de la investigación en las universidades de países subdesarrollados, pone a consideración, crítica y reflexión los "7 pecados capitales de la investigación universitaria Tercermundista": "La desarticulación y fragmentación; El Individualismo; Investigar por investigar; Dependencia y colonialismo (imitación, réplica y subordinación); Orfandad epistemológica (la falta de raíces y de visiones amplias); Desconexión de las demás Funciones Universitarias; El dominio de la Burocracia (la inteligencia controlada por el analfabetismo poderoso)" (pp. 1-12); descripción no lejana a la realidad de universidades como es el caso de Bolivia.

A planteamientos como los citados se unen posiciones y normativas académicas que centran la atención en el desarrollo de la metodología de la investigación -como asignatura y como procedimiento unívococomo punto de partida para desarrollar ciertos procesos investigativos, recetas predefinidas que deben ser seguidas, en algunos casos carecen de razonamiento de teorización, esto se convirtió en uno de los obstáculos el aprendizaje de la investigación.

Desde los razonamientos planteados de Mario Bunge sobre el uso del término metodología de la investigación, resulta contraproductiva siempre que sea concebida como una unidad curricular en el ámbito del aula como una especie de manual. De lo que se trata es de concebir la metodología como proceso filosófico asumirla como operaciones de investigación apoyada en una teoría especial de investigación. Por ello, debe ser convertida en tecnologías de investigación lo que va a presuponer una teoría de investigación preconcebida.

Descubrir el estilo de pensamiento que cada investigador asume deberá ser potenciado des- de sus propias configuraciones cognitivistas. De ahí están aquellos que tienden a desarrollar el estudio observando los hechos este tipo de investigadores podrán ser entrenados desde la teoría empirista inductivista, por otro lado a quienes tienen configuraciones cognitivistas que tienden a hacer empatía, parten de sus experiencias que desarrollan, se debería entrenar en una teoría vivencialista, una configuración cognitiva desde una posición cognitivista racionalista deductivista deberá ser potenciada con tecnologías racionalistas deductivistas.

De esta concepción metodologista, los investigadores en formación reducen sus posibilidades de aprender a investigar asumiendo manuales a seguir sin que encuentren motivación, comprensión o interés auténtico por la investigación; se limitan a conocer, describir, explicar la investigación, más no a generar investigación. De ahí la importancia de asumir la didáctica superior de la investigación mediante estrategias muy bien reflexionadas y responsablemente aplicadas en el contexto formativo de los nóveles investigadores a partir de la preparación, conocimiento y experiencia propia del docente en procesos investigativos para tener mayores luces a la hora de enseñar a investigar.

\section{Tecnología didáctica para la formación de investigadores}

La tecnología didáctica connota, especial importancia en la formación de investigadores. Su componente esencial son las estrategias de enseñanza desafiadas a ser los medios más concretos que deben ser trabajados con especial cuidado para obtener los resultados en la enseñanza y el aprendizaje de la investigación. 
En la perspectiva de Chirino-Ramos (2012) la didáctica aporta, desde sus fundamentos teóricometodológicos, los recursos para llevar a la práctica educativa esta formación desde las diferentes disciplinas y asignaturas del currículo. Considerar la didáctica como compo- nente fundamental del proceso de enseñanza aprendizaje de la investigación, para la autora implica:

Participar en la construcción del conocimiento científico

- Generar habilidades científico - investigativas, para ello se debe:

- Problematizar la realidad educativa

- Teorizar la realidad educativa

- Comprobar la realidad educativa

- Asumir valores ético profesionales

- Considerar el método y los medios de enseñanza - aprendizaje

- Formas de organización y la evaluación.

Elementos que visibilizan la importancia del uso de la didáctica en la enseñanza de la investigación en tanto de ella depende la producción de conocimiento que en la perspectiva diacrónica de la investigación, no solo describa los hechos, sino que avance a la fase de explicación, supere la fase contrastiva para llegar a la fase aplicativa. Concebido así, el proceso de producción aportará a la generación de conocimiento que transforme los hechos y situaciones de la vida cotidiana.

De ahí la importancia que debe asignársele a los procesos de enseñanza, de la didáctica en este campo particular de la educación en las aulas universitarias de contextos latinoameri- canos. La didáctica de la investigación estará conformada por un conjunto de estrategias de enseñanza propias y muy bien definidas. El Dr. Calderón (2018), parte por definir a las estrategias como un grupo de acciones técnicas y procedimentales que se realizan a un corto, mediano y largo plazo. Se debe, entonces de hablar de estrategias a partir de las etapas de manera particular dentro de todo el proceso investigativo.

La tecnología didáctica en la formación de investigadores en la perspectiva de Fernández (2018) implica asumir una posición clara respecto a los escenarios, teóricos, contextuales y metodológicos que entran a ser parte del proceso formativo. Implica, entonces una serie de consideraciones válidas y necesarias en su desarrollo:

- La clarificación conceptual como estrategia que favorece la formación de investiga- dores, por cuanto permite comprender el punto de vista de cada quien y en conse- cuencia la percepción que se tiene de la realidad.

- La configuración del contexto investigativo, a partir de cómo se concibe la investi- gación y en qué contexto o realidad concreta se desarrolla.

- La investigación y la educación se ven articuladas a partir de la tecnología didáctica para la formación de investigadores.

- Como enseñar a investigar empleando la diversidad de posibilidades que las tec- nologías didácticas brindan, diseñando incluso nuevas formas de enseñar, nuevas tecnologías didácticas.

- Desarrollar un cúmulo de competencias que el investigador debe poseer: lecturas críticas de la realidad, generar propias interpretaciones, actitudes superadoras de lo metodológico, genera conocimiento, promueve la generación de investigadores honestos, responsables, libres y críticos.

La enseñanza de la investigación, por tanto, implica contextualizarnos en el ámbito de la educación mediada por las tecnologías didácticas que permiten la formación de investigadores que más allá de conocer teorías, explicar conceptos, analizar o interpretar realidades, puedan generar conocimiento científico en tanto posea tres propiedades: socialización que tenga repercusión social, masiva; sistemática repetible, comunicable, 
que conlleve procedimientos, capaz de decir que fue lo que hizo para llegar a respuestas; y fundamento teórico bases teóricas sólidas.

En consecuencia, la tecnología didáctica para la formación de investigadores es un campo importante y que ofrece grandes desafíos cuando de generar conocimiento se trata, conlleva gran expectativa, interés y necesidad de ser discutida como parte de los procesos de enseñanza y de aprendizaje por la demanda creciente de formar investigadores necesarios en las universidades.

\section{RESULTADOS}

La experiencia investigativa en función a los cuatro momentos metodológicamente estable- cidos demostró aspectos diversos en el proceso.

1. Primer momento: Aspectos fundamentales

Inicio el proceso con la planificación y ejecución de un taller donde participaron todos los estudiantes, nóveles investigadores, del tercer nivel de la Carrera de Trabajo Social. En él se dieron las consideraciones generales del proceso a desarrollar. Las estrategias didácticas consistieron en la presentación de imágenes correspondientes a situaciones problemáticas en el ámbito social: pobreza, desnutrición, alcoholismo, violencia, abandono infantil, problemas de vivienda, entre otros; la pregunta ¿qué podemos hacer frente a estas realidades?, fue el detonante para una reflexión general.

La pregunta dio paso a la necesidad de centrar la atención en situaciones concretas libres de ambigüedad y generalizaciones; lo que permitió la incursión a la necesidad de investigar. Ello dio pie a la contextualización de la investigación, la aclaración de algunos conceptos básicos y la motivación en este proceso.

Iniciar un proceso de investigación que permita la vivencia cercana de un hecho o situación que implique un problema social concreto. Fue necesario precisar por cada equipo qué realidades se investigarán, porqué y cuál el propósito de investigar esas realidades.

Finalmente de los 18 estudiantes asignados que participaron se conformaron nueve equipos cuya tarea fundamental fue identificar casos concretos que conlleven determinados proble- mas sociales y con los cuales se pueda desarrollar procesos investigativos desde la investigación vivencialista-intuitiva. Los casos debieron corresponder al entorno del Municipio de Uncía del departamento de Potosí del Estado Plurinacional de Bolivia.

2. Segundo momento: Vivencia, experiencia, relacionamiento

Aproximadamente por más de un mes, los equipos tuvieron la posibilidad de realizar un diagnóstico preliminar a partir del relacionamiento, la cercanía y experiencia con las familias identificadas. Tiempo en el que se obtuvo información en dos direcciones: primero, respecto de la familia y sus características generales como su historia social, grupo familiar, condiciones entre otros; segundo respecto al contexto sociocultural donde se desenvuelven estas familias.

Durante este proceso se abordaron estrategias para la generación de habilidades para el relacionamiento con las familias (normas básicas de convivencia, relaciones humanas); habilidades científicas para la generación de conocimiento como el saber observar, preguntar, analizar e interpretar la información, identificar conceptos, categorías a investigar; y para la generación de actitudes éticas en el proceso de relacionamiento, como el respeto, los valores, reflexionar lo bueno, lo digno.

Los resultados fueron alentadores; cada equipo obtuvo experiencias diversas, casos identificados, un bagaje de información por cada familia, un interesante cúmulo de dudas respecto a cómo enfrentar esas situaciones, se identificaron diversos problemas, se desarrollaron procesos de empatía y cercanía con los casos.

3. Tercer momento: Aspectos teóricos - metodológicos

Las orientaciones didácticas en esta etapa se desarrollaron a partir de un segundo taller, esta vez con base en el conocimiento adquirido a partir del relacionamiento y cercanía obtenida con las familias. La estrategia didáctica asumida fue la identificación de todos los posibles problemas identificados en cada caso, 
la priorización de los principales problemas en función a ciertos criterios (interés de la familia, pertinencia social, impacto disciplinar).

En el mismo taller se trabajó con cada equipo -a partir de su vivencia- en la determinación de la pregunta de investigación, el título, el objetivo y la categorización del problema a investigar, identificando las categorías de estudio. La tarea encomendada fue la precisión de los aspectos metodológicos de la investigación, así como la justificación y contextualización teórica, tarea complementada al cabo de unos días, al presentar el plan de investigación.

La siguiente estrategia didáctica aplicada para la construcción del marco teórico, fue la elaboración de esquemas y mapas conceptuales que permitan identificar las principales ideas acerca el tema por cada categoría del problema a investigar. Cada idea se convierte en un aspecto a desarrollar partiendo de su precisión conceptual, abordaje teórico, comparación e interpretación del estudiante. Se manejaron citas y sus respectivas reflexiones o posturas del estudiante.

En la etapa de acopio de información la relación emprendida con las familias, fue fundamental, ello permitió la apertura y confianza para la aplicación de los métodos teóricos y empíricos como las historias de vida, la entrevista a profundidad y la observación que fueron empleados en varias ocasiones en función de los temas.

4. Cuarto momento: Orientaciones didácticas para la elaboración del informe de investigación

En la etapa última se realizó la capacitación para la elaboración del informe final de investigación, en donde los estudiantes aprendieron una forma concreta de procesar y presentar resultados de la investigación cualitativa, vivencialista-intuitiva; asimismo respetando las normativas institucionales y académicas establecidas en esta unidad formativa. Los resultados fueron el abordaje y reporte de temáticas diversas, entre ellas: 
TABLA 2.

Temas y categorías de estudio, desde la investigación vivencialista-intuitiva.

\begin{tabular}{|c|c|c|}
\hline \multicolumn{2}{|c|}{$\begin{array}{c}\text { EQUIPOS TÍTULOS DE INFORMES DE } \\
\text { INVESTIGACIÓN }\end{array}$} & $\begin{array}{l}\text { CATEGORIAS } \\
\text { ESTUDIADAS }\end{array}$ \\
\hline $\begin{array}{l}\text { Equipo } \\
1 \text { : }\end{array}$ & $\begin{array}{l}\text { El abandono infantil y sus } \\
\text { repercusiones en el desarrollo } \\
\text { emocional y familiar de los niños. }\end{array}$ & $\begin{array}{l}\text { - Abandono } \\
\text { infantil - } \\
\text { Desarrollo } \\
\text { emocional }\end{array}$ \\
\hline 2: & $\begin{array}{l}\text { El desarrollo psicosocial de los } \\
\text { adolescentes durante el pro-ceso de } \\
\text { separación de los padres. }\end{array}$ & $\begin{array}{l}\text { - Desarrollo } \\
\text { psicosocial - } \\
\text { Adolescente - } \\
\text { Separación }\end{array}$ \\
\hline $\begin{array}{l}\text { Equipo } \\
\text { 3: }\end{array}$ & $\begin{array}{l}\text { Inaccesibilidad a vivienda propia, } \\
\text { como factor determinante para el } \\
\text { desarrollo socioeducativo de las } \\
\text { familias de bajos ingresos } \\
\text { económicos. }\end{array}$ & $\begin{array}{l}\text { - Vivienda - } \\
\text { Desarrollo social } \\
\text { - Bajos ingresos }\end{array}$ \\
\hline $\begin{array}{l}\text { Equipo } \\
4:\end{array}$ & $\begin{array}{l}\text { Características socioculturales en el } \\
\text { matrimonio andino. }\end{array}$ & $\begin{array}{l}\text { - Sociocultural - } \\
\text { Matrimonio - Lo } \\
\text { andino }\end{array}$ \\
\hline $\begin{array}{l}\text { Equipo } \\
5:\end{array}$ & $\begin{array}{l}\text { El desarrollo psicosocial de los hijos } \\
\text { en las familias mono- parentales. }\end{array}$ & $\begin{array}{l}\text { - Familia } \\
\text { monoparental - } \\
\text { Desarrollo } \\
\text { psicosocial }\end{array}$ \\
\hline 6: & $\begin{array}{l}\text { El embarazo en adolescentes y su } \\
\text { influencia en el desarrollo emocional } \\
\text { y el rendimiento educativo. }\end{array}$ & $\begin{array}{l}\text { - Adolescente - } \\
\text { Embarazo precoz } \\
\text { - Desarrollo } \\
\text { emocional - Lo } \\
\text { educativo }\end{array}$ \\
\hline 7: & $\begin{array}{l}\text { Condiciones de habitabilidad e } \\
\text { higiene y su repercusión en la } \\
\text { desnutrición de los niños. }\end{array}$ & $\begin{array}{l}\text { - Habitabilidad - } \\
\text { Higiene - } \\
\text { Desnutrición } \\
\text { infantil }\end{array}$ \\
\hline 8: & $\begin{array}{l}\text { Características socioculturales en } \\
\text { la estructura y roles fami- liares de } \\
\text { la Comunidad de Circuyo - } \\
\text { Municipio de Uncía. }\end{array}$ & $\begin{array}{l}\text { - Socio-Cultural - } \\
\text { Estructura } \\
\text { familiar - Roles } \\
\text { familiares - } \\
\text { Ruralidad }\end{array}$ \\
\hline
\end{tabular}

En base a informes y seguimiento de los procesos Investigativos de estudiantes - investigadores. Carrera de Trabajo Social-Sede Uncía. UATF. Bolivia. 2019

Una estrategia didáctica para generar actitudes éticas y de responsabilidad en el proceso de investigación fue la devolución y socialización de resultados a la población participante, fue la etapa conclusiva y cierre del proceso con cada familia al cabo de un proceso de tres meses de interacción continua.

\section{DISCUSIÓN}

El proceso de investigación encarado generó resultados diversos, el análisis de los mismos puede ser categorizado desde tres perspectivas:

a. En cuanto a los roles: Investigador - Casos/familias - Docente

En el proceso investigativo se visibilizan tres actores fundamentales: las familias identifica- das como casos de estudio; los noveles investigadores; y, la docente como guía que orientó el proceso.

Las familias identificadas tuvieron la característica homogénea de atravesar por problemas sociales lo que desencadenó situaciones complejas, diversas y caracterizadas circunstancial o estructuralmente por su situación de pobreza. Esta condición trajo consigo problemas que generaron desequilibrio en el desarrollo social, emocional, económico y hasta cultural. A partir de sus características propias y de la situación adversa 
que atravesaban, demostraron accesibilidad ante los estudiantes con algunas dificultades al inicio pero que fueron supera- das durante y al finalizar el proceso de investigación. Al cierre del proceso investigativo, la mayoría de las familias presentaron actitudes de gratitud y un relacionamiento positivo ante la presencia de las estudiantes a partir del relacionamiento cercano que sostuvieron.

Los estudiantes investigadores, demostraron interés ante el proceso investigativo, interés que fue incrementando en el momento de relacionamiento con las familias. Obtuvieron experiencia y conocimiento en el campo de la investigación a partir de la cercanía y contacto directo con la población, demostrando responsabilidad, creatividad y criterio propio frente a la investigación cualitativa vivencialista-intuitiva.

Por su parte, el rol del docente fue asumido de una manera seria y comprometida, en un nivel grupal y por equipos de dos personas; velando la comprensión del proceso, orientando en cada etapa, generando las competencias requeridas en el proceso de investigación, reali- zando el seguimiento y supervisión continua.

Tanto para estudiantes como para la docente al ser un proceso nuevo, se encontraron limita- ciones y dificultades como: la ausencia de referentes teóricos respecto a esta tecnología de investigación; la improvisación que en muchos casos fue enfrentada a partir de la intuición de los investigadores; confusión del nivel de empatía que demostraron convertida en procesos asistenciales; excesiva subjetividad. Elementos que deben ser reflexionados pero que desde ningún punto de vista reducen las potencialidades que el proceso brindó.

b. En cuanto a los logros del proceso de investigación vivencialista.

La incursión a procesos investigativos cualitativos desde la investigación vivencialista-intuitiva, fue un espacio novedoso que requirió un seguimiento, capacitación y orientación continua de manera interactiva: estudiante-docente y estudiante-estudiante. Esta experien- cia permitió la inmersión a la realidad concreta de las familias de estudio, encarando los problemas desde la participación de los propios involucrados analizando, descubriendo y generando acciones determinadas que aportaron en cierta medida a la transformación de realidades concretas.

La elaboración de un reporte final como corolario del proceso investigativo, permitió visibilizar resultados concretos en la incursión a la investigación cualitativa desarrollada.

c. En cuanto al proceso de enseñanza y aprendizaje.

Básicamente se generaron habilidades, destrezas y competencias en el ámbito de la investigación cualitativa, a partir de todo el proceso investigativo encarado.

La generación de conocimiento científico, como la abstracción de los fenómenos sociales estudiados, sus características, relaciones que se produjo a partir del acercamiento y relacionamiento con los casos concretos y sobre todo de la comprensión de cada caso.

Lo ético estuvo también presente en el proceder investigativo como la significación social o la cualidad que demostraron los investigadores, lo que orientó la actitud de los estudiantes. La libertad, la responsabilidad, el respeto, la empatía, fueron algunos de los valores éticos generados en el proceso investigativo.

\section{CONCLUSIONES}

La investigación vivencialista-intuitiva, constituye un referente apasionante, para desarrollar investigaciones en los que el investigador se involucra con el sujeto. Genera un relacionamiento empático, ante los hechos y promueve la transformación de realidades concretas. El referente de las cosas exteriores a las cosas interiores y de ellas a las superiores, resume un procedimiento cognoscitivo que toma como base la vivencia y experiencia concreta. Por tanto, la formación de investigadores bajo este referente teórico debe poseer inclinación y decisión para encarar investigaciones en este estilo de pensamiento o enfoque epistemológico.

La formación de investigadores en el campo del trabajo social, encuentra en la investigación vivencialistaintuitivo un proceso alternativo para la enseñanza y el aprendizaje pues permite, no solo conocer sino comprender y transformar la realidad. 
La tecnología didáctica es el nexo idóneo entre la educación y la formación de investigadores. Enseñar a investigar resulta un proceso complejo y diverso en él confluyen concepciones propias de lo que se enseña como procesos investigativos y la forma como se enseña. Estrategias novedosas, creativas, fundamentadas, aplicables será lo que involucre la tecnología didáctica.

\section{REFERENCIAS}

Arteaga, A. (2011). Metodología de la investigación. La Paz: Bolivia.

Calderón, R. (2018). Material audiovisual. Didáctica y su relación con los procesos de investigación. Seminario III. Posdoctorado en Didáctica de la Investigación. Instituto Nacional de Investigación y Capacitación Contínua - Perú.

Chirino-Ramos, M. (2012). Didáctica de la formación inicial investigativa en las universidades de ciencias pedagógicas. La Habana. Cuba.

Fernández, B. (2018). Tecnología didáctica para la formación de investigadores. Seminario IV. Posdoctorado en Didáctica de la Investigación. Instituto Nacional de Investigación y Capacitación Contínua - Perú.

Hernández, A. Comp. (2016). Glosario de "ISMOS” o tendencia en el debate sobre teoría de la investigación.

Padrón Guillén, J. L. (2004). Pecados capitales de la investigación universitaria tercermundista. Informe de Investigaciones Educativas, XVIII.

Padrón, J. (2018). Teoría cognitiva de la investigación científica. Seminario I. Posdoctorado en Didáctica de la Investigación. Instituto Nacional de Investigación y Capacitación Contínua - Perú.

Sánchez, R. (2014). Enseñar a investigar. Una didáctica nueva de la investigación en ciencias sociales y humanas. México: Universidad Nacional Autónoma de México. 